\title{
Successful treatment of AIDS-related pulmonary Kaposi's sarcoma with liposomal daunorubicin
}

\author{
D. Schürmann, A. Dormann, T. Grünewald, B. Ruf
}

\begin{abstract}
Successful treatment of AIDS-related pulmonary Kaposi's sarcoma with liposomal daunorubicin. D. Schürmann, A. Dormann, T. Grünewald, B. Ruf. CERS Journal Ltd 1994.

ABSTRACT: We report a 35 year old male, human immunodeficiency virus (HIV)infected patient with acquired immune deficiency syndrome (AIDS), who presented with rapidly progressive pulmonary Kaposi's sarcoma. The tumour regressed with treatment with liposomal daunorubicin and remained in partial remission until the patient died 12 months later from complications of HIV infection. Liposomal daunorubicin may be a safe and effective alternative to the treatment protocols in current use for pulmonary Kaposi's sarcoma.

Eur Respir J., 1994, 7, 824-825.
\end{abstract}

Pulmonary Kaposi's sarcoma (KS) is a life-threatening complication in patients with the acquired immunodeficiency syndrome (AIDS). The prognosis is poor, with a median survival after diagnosis of 4-10 months [1-3]. The response to chemotherapy is usually limited, and current treatment protocols are associated with considerable toxicity $[3,4]$.

The use of targeted liposomes as a carrier for cytotoxic drugs may reduce the toxic effects on normal tissue, whilst increasing drug concentrations and effectiveness at the tumour site [5]. Recently, it has been reported that liposomal daunorubicin may be a safe and effective formulation for treating AIDS-related KS [6]. Experience with the usefulness of this drug formulation is limited. We report an AIDS patient with rapidly progressive pulmonary KS, who responded to treatment with a liposomal daunorubicin formulation (DaunoXome ${ }^{\circledR}$, Vestar, San Dimas, CA, USA).

\section{Case report}

A human immunodeficiency virus (HIV) seropositive male homosexual patient, aged $35 \mathrm{yrs}$, presented in October 1991 in poor condition, with a 4 week history of cough, dyspnoea and weight loss. HIV infection had been diagnosed in 1985. A right-sided local cutaneous thoracic Kaposi's sarcoma (KS) was diagnosed 14 months earlier, which had temporarily responded to irradiation. The patient had a history of thoracic herpes zoster, recurrent colitis due to Giardia lamblia, and disseminated Mycobacterium avium complex (MAC) infection, the latter having been the initial AIDS diagnosis in March 1990.

The patient had received zidovudine for 15 months. In addition, he was receiving $25 \mathrm{mg}$ pyrimethamine $/ 500 \mathrm{mg}$ 2nd Dept of Internal Medicine (Dept for Infectious Diseases), Rudolf Virchow University Hospital, Freie Universität Berlin, Berlin, Germany.

Correspondence: B. Ruf, 2nd Dept of Internal Medicine, Dept of Infectious Diseases, Rudolf Virchow University Hospital, Augustenburger Platz 1, 13353 Berlin, Germany

Keywords: Acquired immune deficiency syndrome, chemotherapy, pulmonary Kaposi's sarcoma.

Received: August 241993

Accepted after revision November 211993 sulfadoxine (one tablet Fansidar ${ }^{\circledR}$ ), plus $15 \mathrm{mg}$ folinic acid twice a week for prophylaxis against Pneumocystis carinii pneumonia and toxoplasma encephalitis, $200 \mathrm{mg}$ fluconazole twice a week for prevention of oral candidiasis, and $450 \mathrm{mg}$ rifabutin plus $1,600 \mathrm{mg}$ ethambutol daily as maintenance treatment for MAC infection.

Pertinent laboratory values included: CD4+ lymphocyte count $0.003 \times 10^{9} \cdot l^{-1}\left(3 \cdot \mu \mathrm{l}^{-1}\right)$, haemoglobin level 117 $\mathrm{g} \cdot l^{-1}$, platelets $90 \times 10^{9} \cdot l^{-1}$, and leucocyte count $1.7 \times 10^{9} \cdot l^{-1}$ (absolute granulocyte count $1.04 \times 10^{9} \cdot l^{-1}$ ). Chest X-ray revealed a dense infiltration of the lingula (fig. 1). Computed tomography showed extensive reticulonodular perivascular infiltrates in the left lower lobe and lingula. A vascularized tumour was revealed by bronchoscopy, causing a $90 \%$ occlusion of the lingula bronchus. Biopsies were diagnostic of KS. Vital capacity (VC) was reduced to $71 \%$ of the predicted value. Chest X-ray 10 days later showed rapid progression.

Treatment was initiated with $40 \mathrm{mg} \cdot \mathrm{m}^{-2}$ DaunoXome ${ }^{\circledR}$ every 14 days. Dose modification with a dose reduction to $50 \%$ for a granulocyte count less than $1.0 \times 10^{9}$ cells $\cdot l^{-1}$ and with dose delay for 1 week for a granulocyte count less than $0.5 \times 10^{9}$ cells $\cdot l^{-1}$ was planned. Antiretroviral treatment and chemoprophylaxis of opportunistic infections were continued with the same doses and schedules as before chemotherapy.

Respiratory symptoms resolved completely within 4 weeks. VC returned to $110 \%$ of the predicted value after five cycles. Response to treatment was demonstrated by chest X-ray after two cycles. After six cycles, maximal resolution had been achieved, with only small residual alterations being demonstrated by chest X-ray (fig. 2) and thoracic computed tomography. Re-evaluation by bronchoscopy revealed almost complete resolution of endobronchial lesions, with only slightly erythrematous 


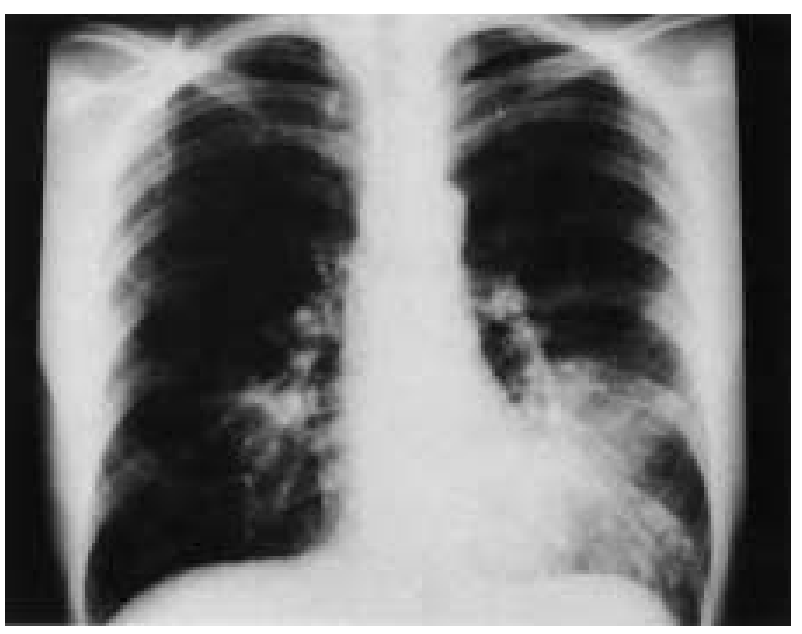

Fig. 1. - Pretreatment chest X-ray showing manifestations of Kaposi's sarcoma in the left lower lobe and lingula.

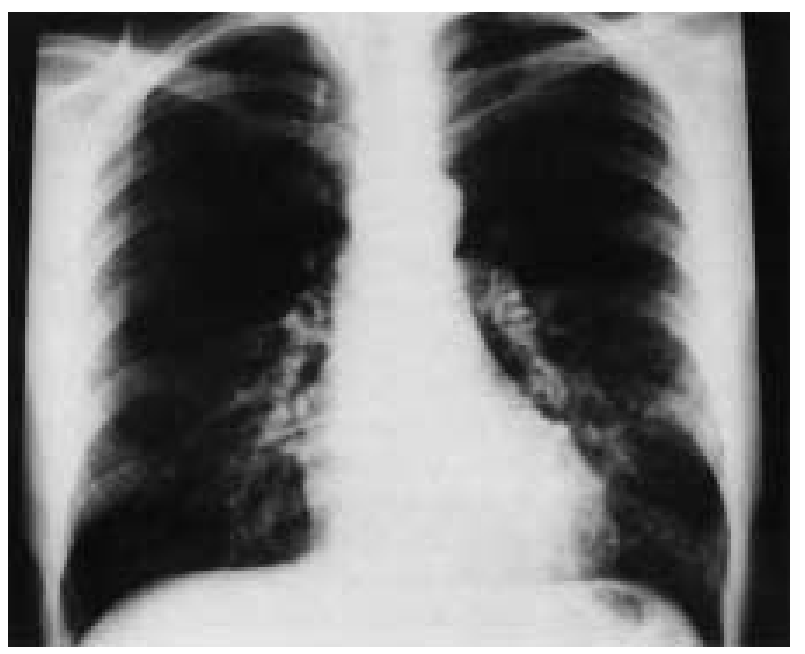

Fig. 2. - Chest X-ray showing maximal response to chemotherapy after six cycles of liposomal daunorubicin.

endobronchial foci being found. Biopsies were negative for KS.

Leucopenia required delay of cycle 3 for 1 week, $50 \%$ dose reduction during cycles 5 and 7, delay of cycle 13 for two weeks, and $50 \%$ dose attenuation for cycles 14-17. After cycle 15, progression was diagnosed, with reappearence of respiratory symptoms and of radiographic manifestations. Granulocyte colony-stimulating factor (G-CSF) (5-10 $\mu \mathrm{g} \cdot \mathrm{kg}^{-1}$ body weight daily) was given between 4-10 days after cycle 17 between all the following cycles, enabling continuation of therapy without dose attenuation. The tumour resolved again, and respiratory symptoms disappeared.

The patient received a total of 21 cycles. Over the last four cycles, the patient developed progressive wasting syndrome and AIDS dementia. One week after the last cycle, he presented with rapid deterioration and died 12 months after the start of chemotherapy with cachexia and pneumonia. Autopsy revealed extensive bacteri- al and cytomegalovirus pneumonia, with only small lesions of Kaposi's sarcoma being present.

\section{Discussion}

The case reported shows that liposomal daunorubicin may be a relatively safe and effective long-term treatment regimen for pulmonary KS. The patient tolerated chemotherapy well for a long duration, in spite of a poor pretreatment condition, advanced AIDS and a markedly reduced bone marrow reserve. The only toxicity seen during the course of treatment was marrow suppression. Importantly, dose reduction required by bone marrow toxicity was associated with progression of KS. However, the use of G-CSF to reduce bone marrow toxicity enabled continuation of the original dose schedule. With continuation of full doses, remission was reinduced and maintained until the patient's death, which was due to progression of his HIV infection.

The efficacy of liposomal daunorubicin in treating AIDSrelated KS, including pulmonary involvement, and its good tolerability has recently been confirmed [7]. The response rate in 25 patients treated with the same formulation of liposomal daunorubicin (DaunoXome ${ }^{\circledR}$ ) as the patient reported here, was $57 \%$. Two of these 25 patients had pulmonary involvement. One patient showed partial remission and the other complete remission of pulmonary KS.

Chemotherapy of AIDS-related KS must be given lifelong, since the tumour progresses rapidly after cessation of treatment $[3,4]$. However, toxicity limits the benefits of chemotherapy, making long-term treatment difficult [3]. Thus, there is need for an effective regimen with low toxicity. Liposomal daunorubicin may be a promising alternative to the chemotherapy protocols in current use.

\section{References}

1. Meduri GU, Stover DE, Lee M, Myskowski PL, Caravelli JF, Zaman MB. Pulmonary Kaposi's sarcoma in the acquired immune deficiency syndrome. Clinical, radiographic and pathologic manifestations. Am J Med 1986; 81: 11-18.

2. Garay SM, Belenko M, Fazzini E, Schinella R. Pulmonary manifestations of Kaposi's sarcoma. Chest 1987; 91: 39-43.

3. Gill PS, Akil B, Colletti P, et al. Pulmonary Kaposi's sarcoma: clinical findings and results of therapy. Am $J$ Med 1989; 87: 57-61.

4. Gill PS, Rarick M, McCutchan JA, et al. Systemic treatment of AIDS-related Kaposi's sarcoma: results of a randomized trial. Am J Med 1991; 90: 427-433.

5. Forsson EA, Coulter DM, Proffitt RT. Selective in vivo localization of daunorubicin small unilamellar vesicles in solid tumors. Cancer Res 1992; 52: 3255-3261.

6. Chew T, Jacobs M, Street H, Crossley R, Ross M. A phase II clinical trial of VS 103 (liposomal daunorubicin) in AIDS-associated Kaposi's sarcoma. In: Poster Abstracts (Abstract PoB 3106), VIIIth International Conference on AIDS/III STD World Congress, Amsterdam, The Netherlands, 19-24 July, 1992.

7. Presant CA, Scolaro M, Kennedy P, et al. Liposomal daunorubicin treatment of HIV-associated Kaposi's sarcoma. Lancet 1993; 341: 1242-1243. 